\title{
Features of Epitaxial Garnet Films for an Atomic Traps Technique
}

\author{
V. N. Berzhansky, V. G. Vishnevskii* , A. S. Nedviga, and A. G. Nesteruk \\ V.I. Vernadsky Taurida National University, Simferopol 95007, Ukraine
}

(Received 3 November 2008, Received in final form 9 July 2009, Accepted 14 July 2009)

\begin{abstract}
High-coercive garnet films have certain parameters suitable for creating reconfigurable magnetic atomic chips with visible geometry. However, the inner stresses and morphological properties, namely, networks of dislocations, layering and surface relief, and dependence of coercivity on thickness must be taken into account. Select features of films important for atomic trap creation have been studied experimentally and the supposed traps concept provided.
\end{abstract}

Keywords : garnet films, coercivity, magneto-optics, atomic traps.

\section{Introduction}

Uniaxial anisotropy high-coercive garnet films (GF) are an optimal medium for thermo-magnetic recording by laser beams or contact printing. For example, contact printing magneto-optic (MO) films have been used [1] for criminalistic examinations of magnetic recordings. Recently [2], it has been established that such GF can be quite promising media for the formation of erasable micro-potentials to assist the trapping of ultra cold neutral atoms $\left({ }^{87} \mathrm{Rb}\right)$. Their infrared transparency and high Faraday rotation in visible light enable manipulation of the atoms and discernment of the reconfigured magnetic trap geometry.

The effective technological approach to increase coercivity $\left(H_{\mathrm{c}}\right)$ is to create hetero-epitaxial strains by increasing the mismatch $\Delta a=\left(a_{\mathrm{f}}-a_{\mathrm{s}}\right)$ between the crystalline lattices of film $a_{\mathrm{f}}$ and substrate $a_{\mathrm{s}}$. Stresses are proportional to the mismatches and networks of misfit dislocations are usually formed for stress relief. As in the case of polycrystalline films [3] that occurred with the aerosol deposition method, imperfect structures due to non-uniform internal stresses are obtained. Strain-induced morphology has a great influence on parameters of monocrystalline GF grown by means of liquid phase epitaxy (LPE). Though known MO traps realizations were successful [4], these circumstances have yet to be taken into account. Thus, contributing to the subject of [5], certain morphological features of strained films are considered herein as both

*Corresponding author: Tel: $+380-652-255198$

Fax:+380-652-517135, e-mail: domain@crimea.edu the formation of the traps and the extent of their localization depend on them.

\section{High-Coercive Garnet Film Synthesis}

Monocrystalline $\mathrm{Gd}_{3} \mathrm{Ga}_{5} \mathrm{O}_{12}$ (GGG) substrates with a (111) crystallographic orientation with only positive substratefilm lattice constants mismatch in a range of: $\Delta a=0.04$ $0.12 \AA$ were employed. After mechanical polishing with diamond abrasives (with an optical quality surface) and ion beam etching, the final thickness of the GF was $h=$ 1.0-2.0 $\mu \mathrm{m}$. As a rule, this processing increases GF coercivity $[1,5]$.

According to the GF application, saturation magnetization $4 \pi M_{\mathrm{s}}$ value of the films must be as high as possible and, additionally, off times it is necessary to provide a Curie point value of $T_{C}>200^{\circ} \mathrm{C}$. Thus, the films of $(\mathrm{Bi}$, $\mathrm{Sm}, \mathrm{Lu})_{3}(\mathrm{Fe}, \mathrm{Al}, \mathrm{Ga})_{5} \mathrm{O}_{12}$ and $(\mathrm{Bi}, \mathrm{Sm}, \mathrm{Lu})_{3}(\mathrm{Fe}, \mathrm{Al}, \mathrm{Sc})_{5} \mathrm{O}_{12}$ were synthesized on GGG substrates with a lattice parameter of $12.383 \AA$, using the isothermal dipping technique.

Melt compositions, defined by the parametric convention of Blank-Nielsen [6], were as follows:

for the variant of $(\mathrm{Bi}, \mathrm{Lu}, \mathrm{Sm})_{3}(\mathrm{Fe}, \mathrm{Al}, \mathrm{Ga})_{5} \mathrm{O}_{12}$ :

$\mathrm{R}_{1}=\mathrm{Fe}_{2} \mathrm{O}_{3} / \mathrm{Ln}_{2} \mathrm{O}_{3}=6.36-7.24 ; \quad \mathrm{R}_{2}=\mathrm{Fe}_{2} \mathrm{O}_{3} / \mathrm{Me}_{2} \mathrm{O}_{3}=10-19 ;$ $\mathrm{R}_{4}=\Sigma$ Garnet $/ \Sigma$ Melt $=0.057-0.073$;

for the variant of $(\mathrm{Bi}, \mathrm{Sm}, \mathrm{Lu})_{3}(\mathrm{Fe}, \mathrm{Al}, \mathrm{Sc})_{5} \mathrm{O}_{12}$ :

$\mathrm{R}_{1}=10-15 ; \mathrm{R}_{2}=10-20 ; \mathrm{R}_{4}=0.09-0.1$.

For both compositions $\mathrm{R}_{3}=\mathrm{PbO} / \mathrm{Bi}_{2} \mathrm{O}_{3} \approx 2$.

$\mathrm{Ln}_{2} \mathrm{O}_{3}$ is the sum of rare-earth oxides and the solvent oxides are $\mathrm{PbO}$; for $\mathrm{Me}_{2} \mathrm{O}_{3}, \mathrm{Me}$ are $\mathrm{Al}, \mathrm{Ga}$, and $\mathrm{Sc}$ ions or combinations thereof. In the numerator position, $\mathrm{R}_{4}$ is the 

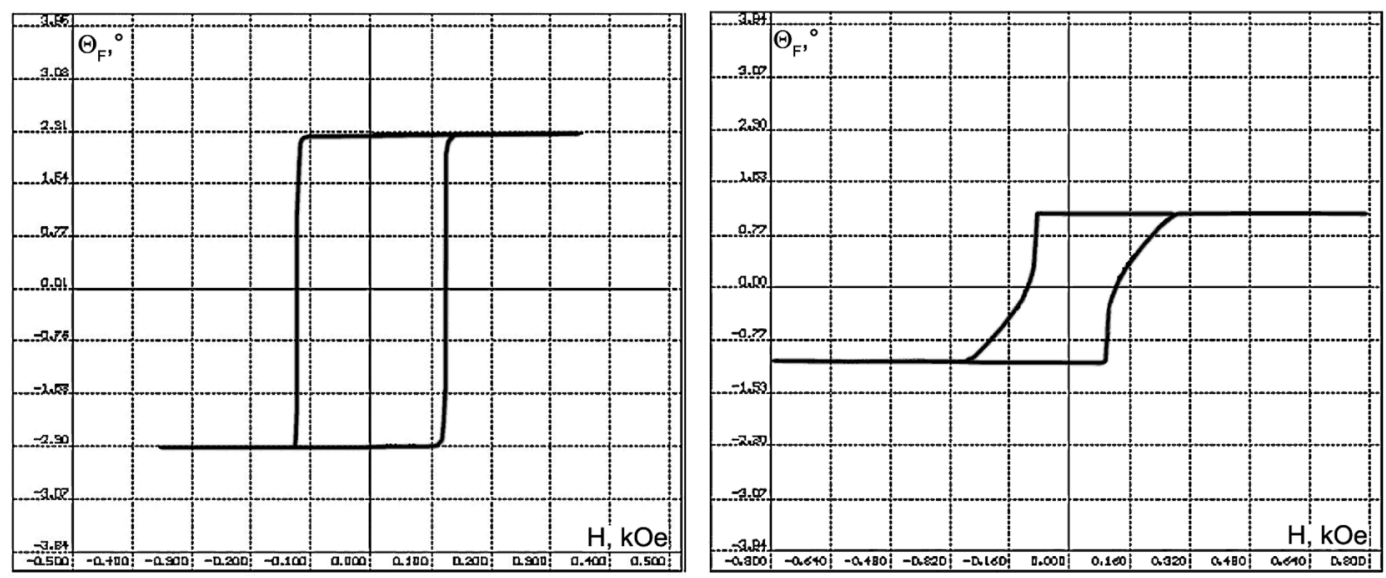

Fig. 1. MO loops of typical GF samples (magnetopolarimeter; light wavelength $\lambda_{1}=0.63 \mu \mathrm{m}$ ).

sum content of all garnet-forming components, while in the denominator position, it is the sum content of all oxides, including those that are garnet-forming. The oxide contents are presented in molar parts.

As a result, as-grown films possessed: $H_{\mathrm{c}}=80-550$ Oe; $\theta=2.0-2.3 \%$ m $\left(\lambda_{1}=532 \mathrm{~nm}\right) ; 4 \pi M_{\mathrm{s}}>1.0 \mathrm{kGs}$; uniaxial anisotropy field $H_{k}=1.5-2 \mathrm{kOe} ; h=4.0-5.0 \mu \mathrm{m} ; T_{\mathrm{C}}=190-$ $210^{\circ} \mathrm{C}$. Fig. 1 presents the MO hysteresis loops of samples at different ratios of coercivity to nucleation magnetic field.

\section{Morphological and Functional Properties}

Figure 2 demonstrates the initial morphology ("faceting") of a typical GF sample (Nomarskii DIC-differential-interference contrast) and MO images of two types of domain structures (DS) obtained after demagnetizing the same film's area by a variable magnetic field (stable DS) and heating to $T>T_{\mathrm{C}}$ (meta-stable DS). Both have fractal signs though they maintained a hexagonal symmetry conditioned by residual cubic anisotropy in the (111) plane. Fractal dimensionality of the meta-stable DS was higher. The existing two types of DS (hysteresis) are standard properties of strained GF [7].
The "faceting" (mosaic of dimples) characteristic of the highly compressed GF contour after LPE is formed by the intersection of dislocations with its surface. The dimples are the points of emergence of dislocation half-loops, which bore misfit segments parallel to the interface [8]. These segments relieve compressive stresses while those of a thread-like nature perturb the growth of the remaining film sufficiently to result in a "cellular" morphology. The extent of the modifications depends upon $\Delta a$ (Fig. 3 ).

To investigate dislocation distribution, some of the samples were polished obliquely; slanting wedges were formed and selectively etched with $\mathrm{H}_{3} \mathrm{PO}_{4}\left(100^{\circ} \mathrm{C}, 1 \mathrm{~min}\right)$ and $\mathrm{HNO}_{3}$ $\left(70^{\circ} \mathrm{C}, 15 \mathrm{~min}\right)$. It has been established [5] that the misfit dislocation network is located above the substrate-film interface and that its position depends on the value of $\Delta a$. At larger mismatches, the height of occurrence of the first dislocations above the interface tended to be smaller, indicating that the pseudomorphic layer, within which the elastic stresses are mainly concentrated, had a certain thickness value.

To explain this tendency, an attempt at composition variation determination in the GF sub-layers was undertaken. Energy dispersive X-ray microanalysis (EDS) after stepped (one sub-layer at a time) ion-beam etching of the
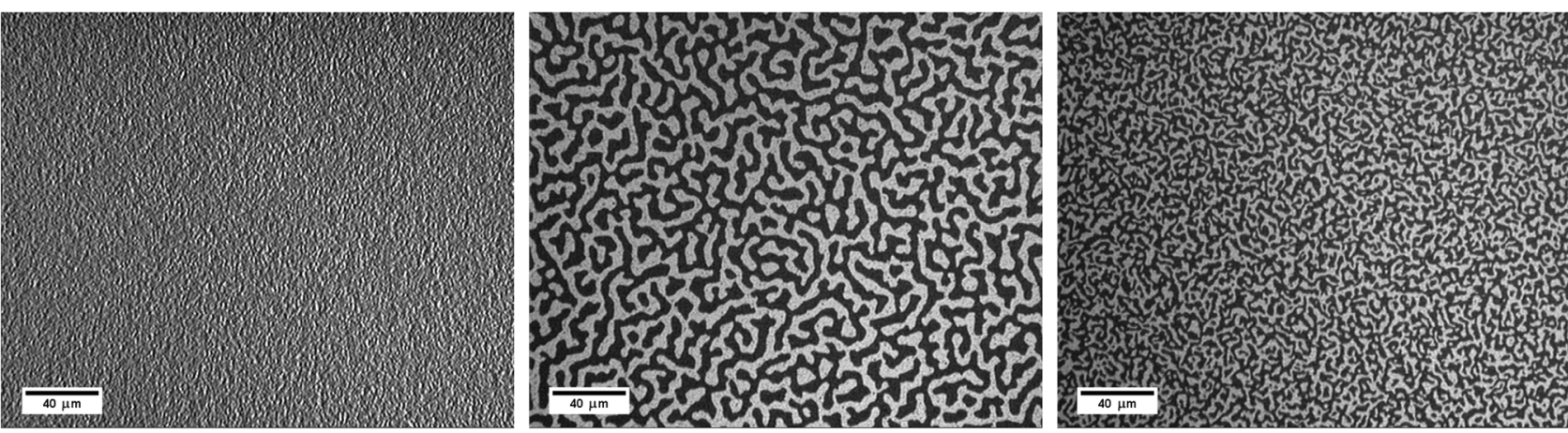

Fig. 2. From left to right: GF's faceting (DIC), stable and meta-stable DS (polarized light). 

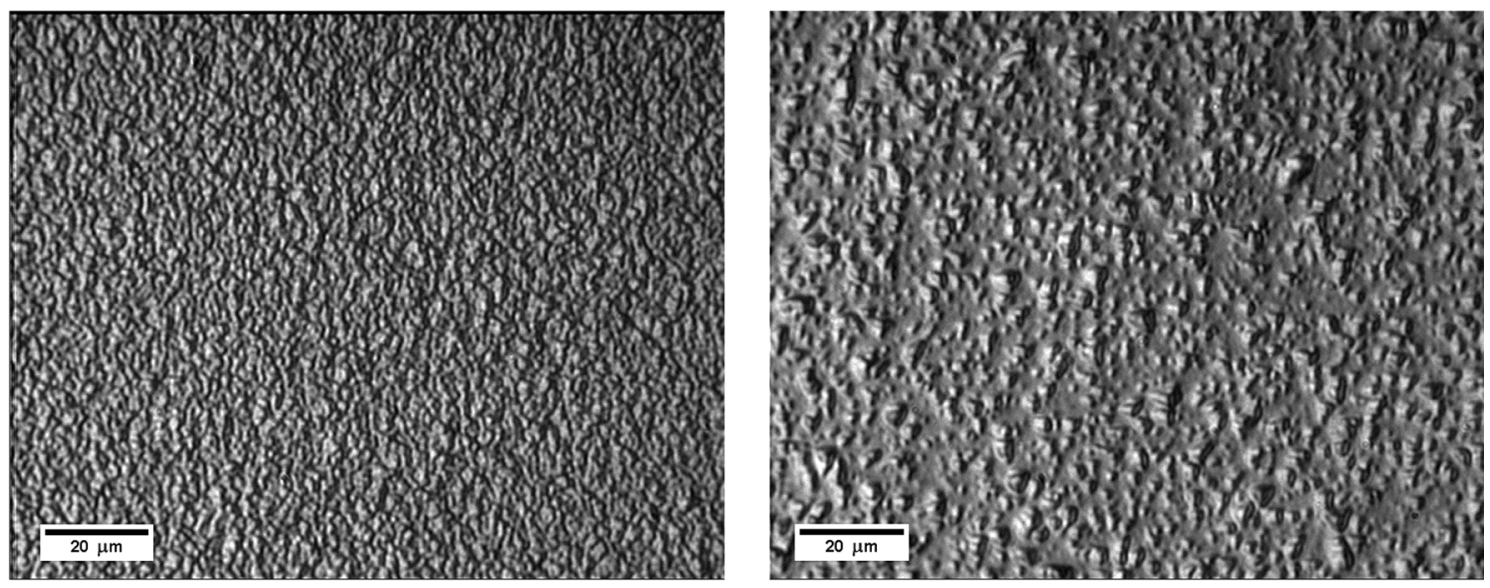

Fig. 3. Films "faceting": $\Delta a=0.084$ (left) and $\Delta a=0.103$ (right). DIC.

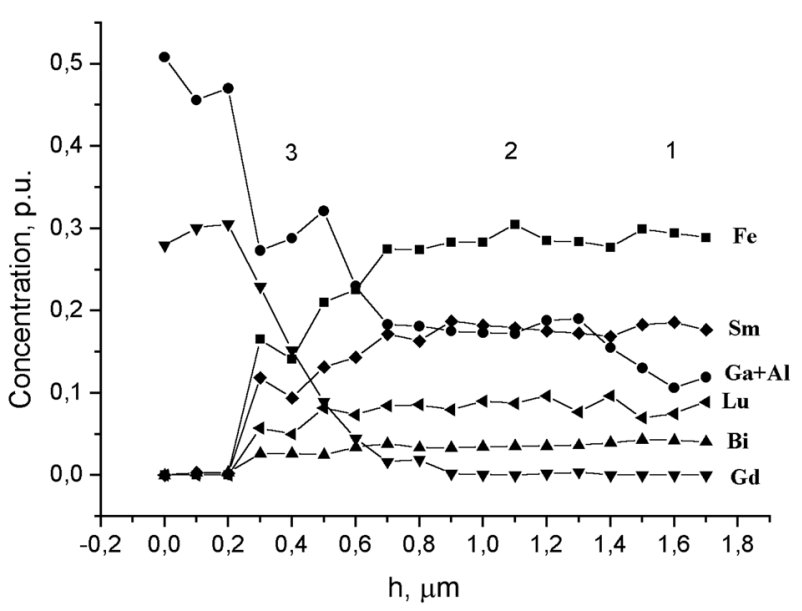

Fig. 4. One sub-layer at a time distribution (in per-unit) of elements in the film sample.

GF sample was used. The experiment confirmed (Fig. 4) the composition stratification according to which the closest to GGG film sub-layer (zone No $3, \mathrm{~h}<0.8 \mu \mathrm{m}$ ) was enriched in $\mathrm{Ga}, \mathrm{Al}$, and $\mathrm{Gd}$ ions, in spite of upper layers (zones No 1 and No 2), in Me ions (Fe, Sm, Lu). The relatively high Gd contribution (zone No 3) can be explained both by the diffusion from the substrate and the depth of probing (estimation of EDS penetration depth is $1.0 \mu \mathrm{m})$. Nevertheless, this indicated that the $\mathrm{T}_{\mathrm{C}}$ value of the upper sub-layers must be higher.

Thus, the hypothesis of Miller and Caruso [8] was verified experimentally through confirmation that when a substrate is dipped into a melt, the first material to grow depletes the melt adjacent to the interface of those constituents, enabling its lattice parameter to approach that of the GGG. Then as the GF grows, the lattice parameter of the layer approached an equilibrium composition of the melt and surpassed the mismatch stress necessary for dislocation generation.
In addition, the influence of the mismatch $\Delta a$ (measured by X-ray diffractometry) on the behavior of coercivity in the GF was studied. As $\Delta a$ increased, $H_{\mathrm{c}}$ initially grew, passed through a maximum, and then decreased [9]. The maximum was related to the periodic localized stresses caused by misfit dislocations networks with periods comparable to the width of domain walls. The period of localized stresses was determined by $\Delta a$. Thus, it was possible to predict the $H_{\mathrm{c}}$ level in a comparatively broad range of mismatches. Furthermore, decreasing $H_{\mathrm{c}}$ at high $\Delta a$ values could be explained by $100 \%$ stress relief due to generation of the misfit dislocations network [8].

"Cellular"-like GF structures limit trap localization. Thin recorded lines must have zigzag binding. However, it is the contention of the authors that the non-uniform stress distribution and, moreover, composition stratification along the growth direction, can be used to create specialty types of traps.

\section{Potential Possibilities of Layered Garnet Films for Multilevel Traps}

Trapping potentials for ultra cold atoms can be formed as the superposition of an external field and stray fields from recorded domain patterns (Fig. 5). For example, the combination of a cylindrical domain stray field with a uniform magnetic field can create a quadrupole-type trap $[2]$.

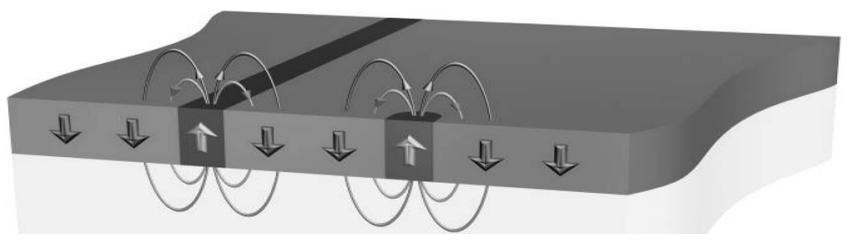

Fig. 5. Stray fields above the recorded domain patterns in monolayer GF. 

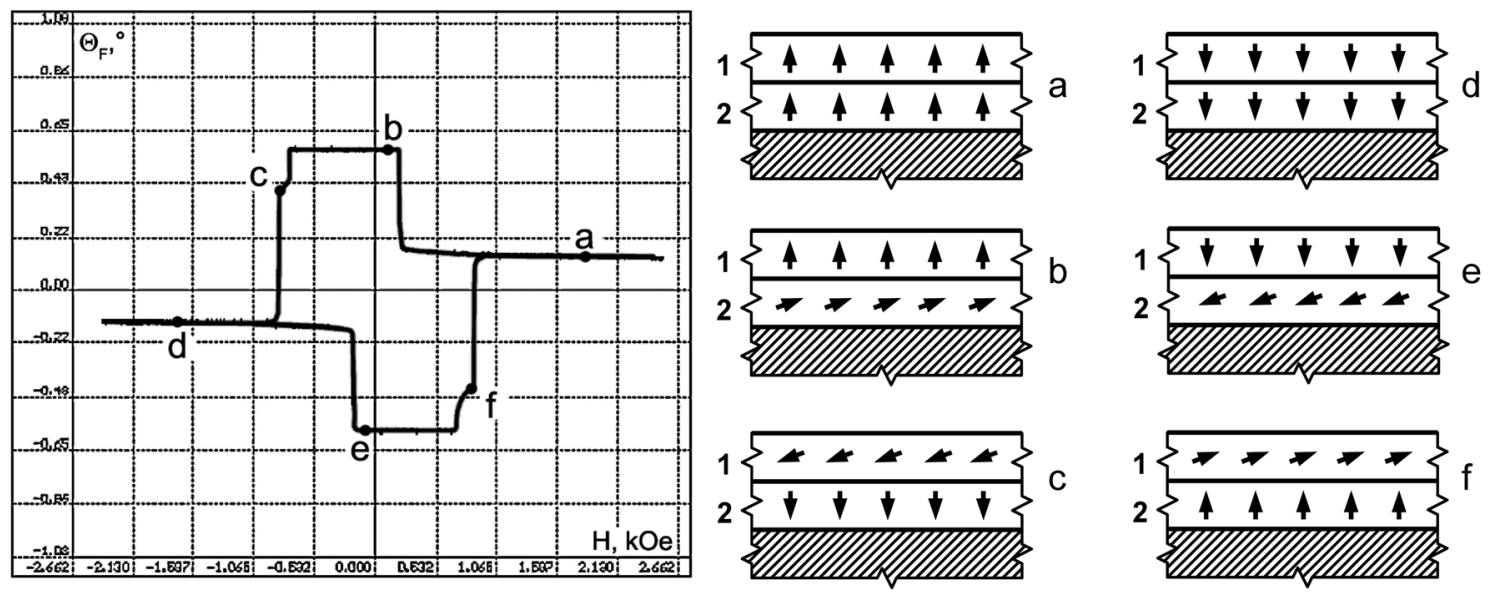

Fig. 6. MO loop of double-layered film (left) and magnetization switching (right).

Due to a high $H_{\mathrm{c}}$ value, the domain pattern (like a permanent micro-magnet) can be stable within a certain range of changing homogenous external fields. Thus, the local stray field, and thereby the trap "level", are practically constant. However, in the case of double-layered film, it is possible to operate the trap "level" in order to localize atom clouds at different (but fixed) distances from the garnet surface. It is trapping in a " $3 \mathrm{~d}$ "-like manner. The principal possibility of layered GF synthesis is defined in Fig. 6.

This experimental MO hysteresis loop demonstrates more than two stable magnetic states that can be used for trap "level" operation. Taking into account left and right Faraday rotation in adjacent layers, corresponding variants of magnetization switching can be schematically shown in Fig. 6. However, independent thermo-recording of domain patterns in the adjacent layers could be provided only through technological selection of certain values for $H_{\mathrm{c}}$, $4 \pi M_{\mathrm{s}}$ and firstly, $T_{\mathrm{C}}$. It is thus necessary to create a sharp gradient of properties along the film thickness.

It is known [10] that during the LPE process the melt temperature determines the segregation coefficients of all garnet-forming elements. If the growth temperature decreases, then a decrease in Me-ion $(\mathrm{Ga}, \mathrm{Sc}$, or $\mathrm{Al}$ in the case herein) concentration and an increase in Bi-content in garnet are obtained (in comparison to initial growth temperature conditions). The value and the regularity of temperature changing at LPE were chosen with the objective of obtaining appreciable differences in parameters, but with nearly equal thickness in GF sub-layers. This way, composition stratification was enlarged and doublelayered GF samples were synthesized experimentally.

Features of thermo-recording can be clarified from Fig. 6 and 7. Initial conditions are as follows: $H_{\mathrm{ci}} M_{\mathrm{si}} ; T_{\mathrm{C} 1}>$ $T_{\mathrm{C} 2} ; M_{\mathrm{s} 1}>M_{\mathrm{s} 2} ; H_{\mathrm{c} 1}<H_{\mathrm{c} 2}$. Pre-recording can include ap- plication of a homogenous external field, $|H|>H_{\mathrm{c} 2}$ only. Absence of local heating (Fig. 6(a, d)) is a variant (1), another is stepped (2). At the beginning the field, $H>H_{\mathrm{c} 2}$, and then the reversed fields $H_{\mathrm{c} 1}<|-H|<H_{\mathrm{c} 2}$ are applied. Fig. 7(d, e) demonstrates the corresponding states of magnetization.

Recording of the through-type domain pattern after prerecording (1) foresees local heating up to $T>T_{\mathrm{C} 1}$ and the simultaneous application of a reversed field $|-H|<H_{\mathrm{c} 1}$ (Fig. 7(a)). Recording of the "bottom" domain pattern after step (1) is realized by local heating up to $T_{\mathrm{C} 2}<T<$ $T_{\mathrm{C} 1}$ in the presence of a reversed field $M_{\mathrm{s} 10}<|-H|<H_{\mathrm{c} 1}$
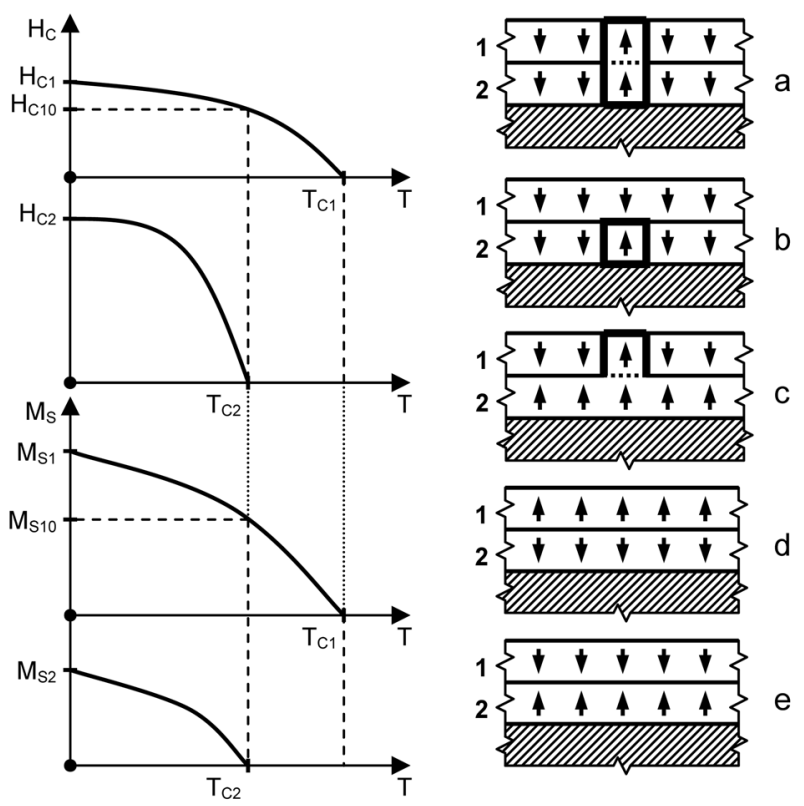

Fig. 7. Features of thermo-recording in double-layered GF. Left - dependences of coercivity and magnetization of GF sublayers upon temperature; right-magnetization switching and recorded patterns. 

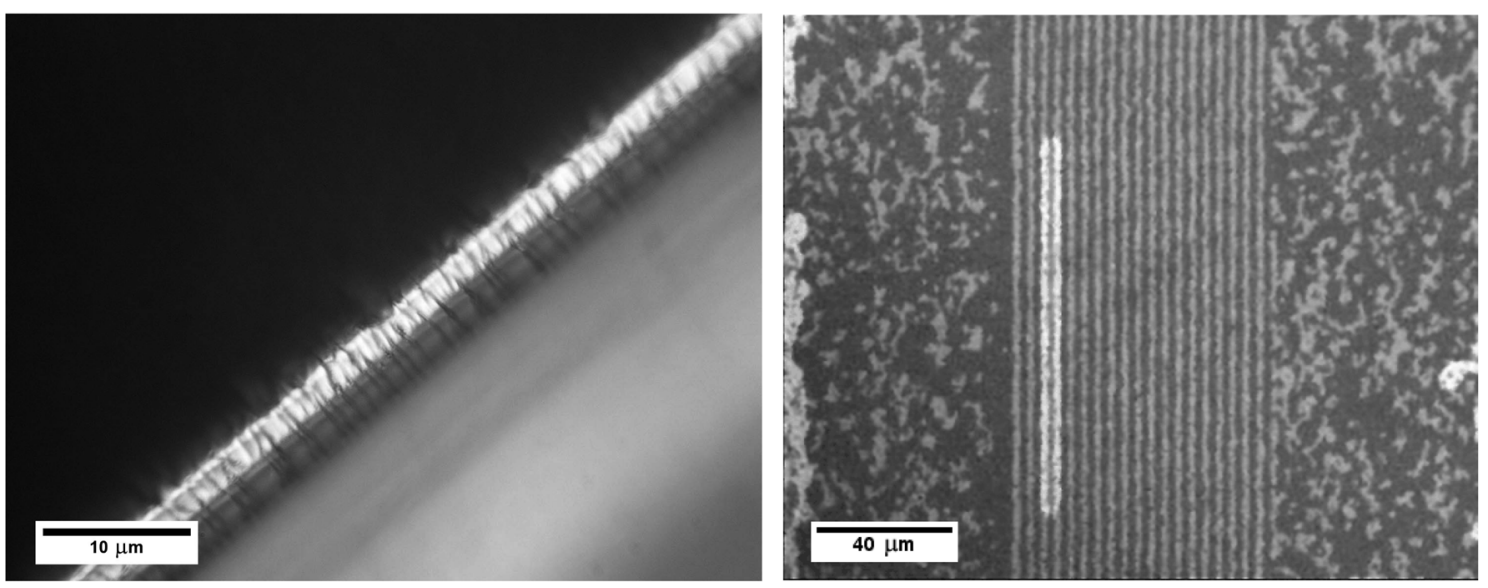

Fig. 8. Double-layered film's edge (left) and thermo-recorded domain patterns (right).

(Fig. 7(b)). Lastly, recording of the "top" domain pattern after pre-recording in accordance to variant (2) can be made by local heating up to $T>T_{\mathrm{C} 1}$, even if the reversed field $|-H|=0$ (Fig. 7(c)).

Fig. 8 presents the as-grown film edge (DIC) and domain patterns (polarized light) recorded as $2.0-\mu \mathrm{m}$-width parallel (vertical) lines in the top layer of the film; additional local switching of the bottom layer is seen as a narrow portion of the image with enhanced brightness.

In practice, the atomic chip realizations now foresee preliminary thermo-recording of the MO trap out of a vacuum chamber $[2,4]$. However, in principle, the chip installation can be supplied with attachment for in situ recording by a through-the-window focusable laser beam.

Possibilities of atomic traps drawing in the GF by focused laser beam were investigated with a setup (Fig. 9) slightly different from scheme [2]. The setup components were as follows: 1 - laser $(532 \mathrm{~nm}) ; 2-\lambda / 2$ plate; 3 electro-optic modulator; 4 - $\lambda / 4$ plate; 5 - 9-polarizers; 6 beamsplitter; 7 - objective; 8 - x-y micro-stage with movement controller 17; 10 - blue LED array; 11 - magnetic field coil; 12 - DC current controller; 13 - bandpass filter; 14 - analyzer; 15 - projection lens; 16 - CCD.

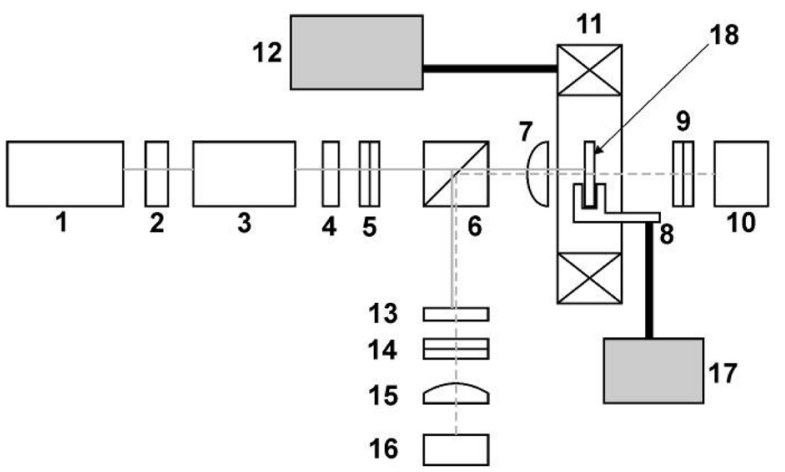

Lines and dots (Fig. 9) having a width of 2.0-5.0 $\mu \mathrm{m}$ were recorded under conditions of $10 \mathrm{~mW}$ power laser beam pulses (20-30 $\mu \mathrm{s})$ and a reverse magnetic bias field of $\sim 0.1 H_{\mathrm{c}}$. Small zigzag-type modifications of the recorded lines width were consequences of dislocation networks in the strained GF. As such, the trap geometry strongly depended on the morphological GF properties; dislocation density determined the limit of the trap dimensions. The average value of the density was $10^{8} \mathrm{~cm}^{-2}[5,8]$. As this density depended on $h$ at a fixed $\Delta a$, extra-thin films were used so that at least the equilibrium dimension of the stable domains increased.

\section{Conclusions}

It is the contention of the authors that new experiments in high-coercive film growth must be oriented to provide "multi-level" atomic traps and traps having dimensions in the sub-micron range. These tasks call for nanotechnology, but the existence of the dislocation networks prevents the required development. Officially, new GF for these purposes should be the result of a compromise in a couple of the parameters.

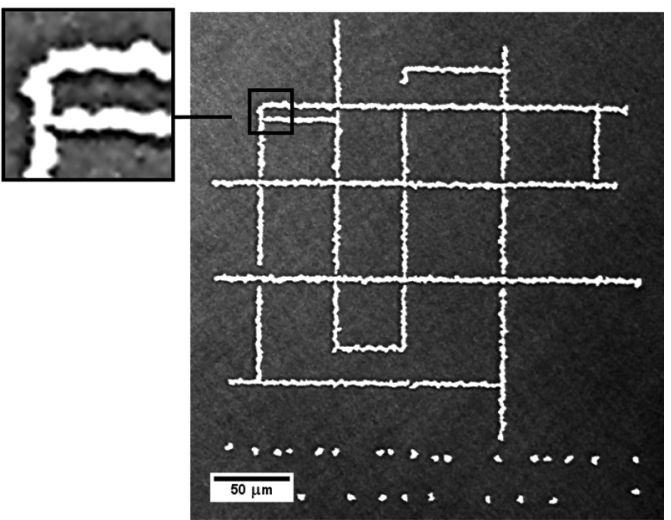

Fig. 9. Experimental setup for $\mathrm{MO}$ recording (left) and recorded lines and dots (right). 


\section{References}

[1] V. Vishnevskii, A. Nesteruk, A. Nedviga, S. Dubinko, and A. Prokopov, Sens. Lett. 5(1), 29 (2007).

[2] A. Jaakkola, A. Shevchenko, K. Lindfors, M. Hautakorpi, E. Il'yashenko, T. Johansen, and M. Kaivola, Eur. Phys. J. D. 35(1), 81 (2005).

[3] K.-H. Shin, M. Mizoguchi, and M. Inoue, J. Magnetics 12, 129 (2007).

[4] A. Shevchenko, M. Heilio, T. Lindvall, A. Jaakkola, I. Tittonen, and M. Kaivola, ArXiv: Quantum Physics/0601163 (2006).

[5] V. Berzhansky, A. Nedviga, V. Vishnevskii, and A.
Prokopov, Solid State Phenomena 152-153, 11 (2009).

[6] D. Gualtieri and P. Tumelti, J. Appl. Phys. 57(8), 3879 (1985).

[7] R. Mikherskii, S. Dubinko, V. Vishnevskii, A. Nedviga, and A. Prokopov, Ukrainian J. Phys. (Ukraine) 45(3), 368 (2000).

[8] D. Miller and R. Caruso, J. Cryst. Growth 27, 274 (1974).

[9] S. Dubinko, A. Nedviga, V. Vishnevskii, A. Shaposhnikov, V. Yagupov, A. Nesteruk, and A. Prokopov, Tech. Phys. Lett. 31(11), 979 (2005).

[10] W. Tolksdorf and C.-P. Klages, Thin Solid Films 114 (12), 33 (1984). 